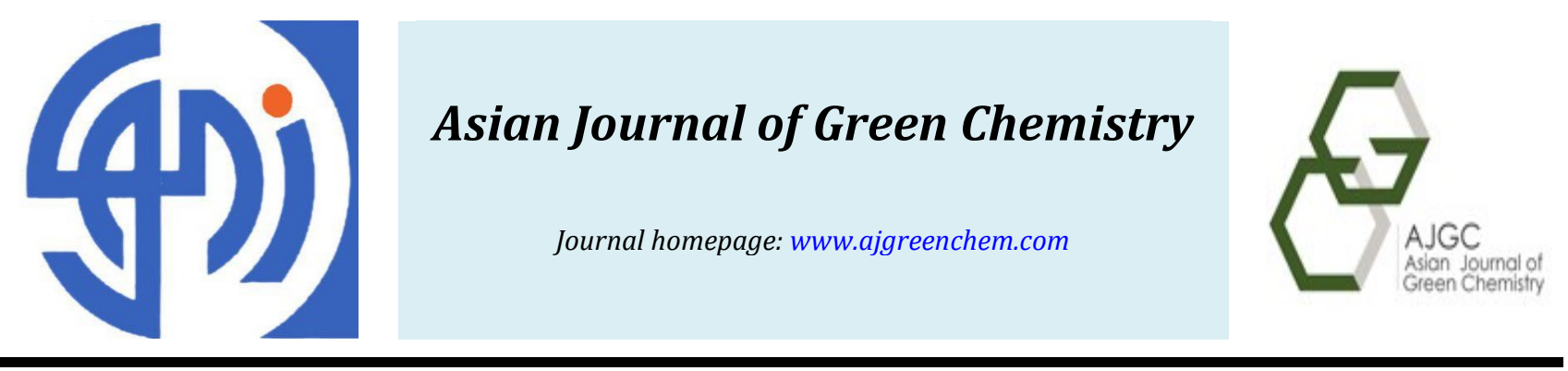

Original Research Article

\title{
Green synthesis and antibacterial activity of cadmium sulfide nanoparticles (CdSNPs) using Panicum sarmentosum
}

\author{
Irshad Ul Haq Bhat*, Yong Sin Yi \\ Faculty of Earth Science, Universiti Malaysia Kelantan, Campus Jeli, 17600, Kelantan, Malaysia
}

\section{ARTICLE INFORMATION}

Received: 28 August 2018

Received in revised: 9 September 2018

Accepted: 28 October 2018

Available online: 17 January 2019

DOI: 10.33945/SAMI/AJGC.2019.4.3

\section{KEYWORDS}

Scanning electron microscopy

X-ray fluorescence

Thermal gravimetric analysis

\begin{abstract}
The plant sources can act as potential precursors for the synthesis of nanoparticles in non-hazardous ways as plants contain various secondary metabolites, acting as reducing and stabilizing agents for the reduction reaction to synthesize novel metallic nanoparticles. The green synthesized nanoparticles have been proven to control various diseases with less adverse effect. Thus, in this study, the green method for preparation of cadmium sulfide nanoparticles using Panicum sarmentosum was adopted. The synthesized CdSNPs were evaluated for their optical, structural, surface morphological, and antibacterial properties. The CdSNPs were characterized using UV-vis spectrophotometry, Fourier transmission infrared spectroscopy (FT-IR), X-ray diffraction analysis (XRD), scanning electron microscopy (SEM), X-ray fluorescence (XRF), and thermal gravimetric analysis (TGA). The antibacterial activity against Staphylococcus aureus and Escherichia coli was also carried out. The XRD pattern revealed the crystalline structure of CdSNPs. The SEM analysis showed the size and shape of the nanoparticles. XRF analysis confirmed the presence of cadmium and sulphur in the nanoparticles. The presence of $(\mathrm{OH}),(\mathrm{NH})$, and carboxylic functional groups were confirmed by the FTIR analysis. TGA results prove that the CdSNPs are more thermally stable than the plant material. The ecological friendly methods can generate simple, easy, and cost-effective nanoparticles compared with the chemical and physical approaches, and have a potential to be used as antibacterial agents.

(C) 2019 by SPC (Sami Publishing Company), Asian Journal of Green Chemistry, Reproduction is permitted for noncommercial purposes.
\end{abstract}




\section{Graphical Abstract}

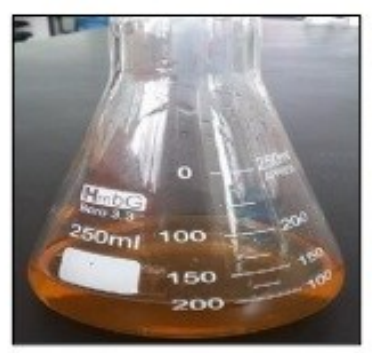

Nanoparticles
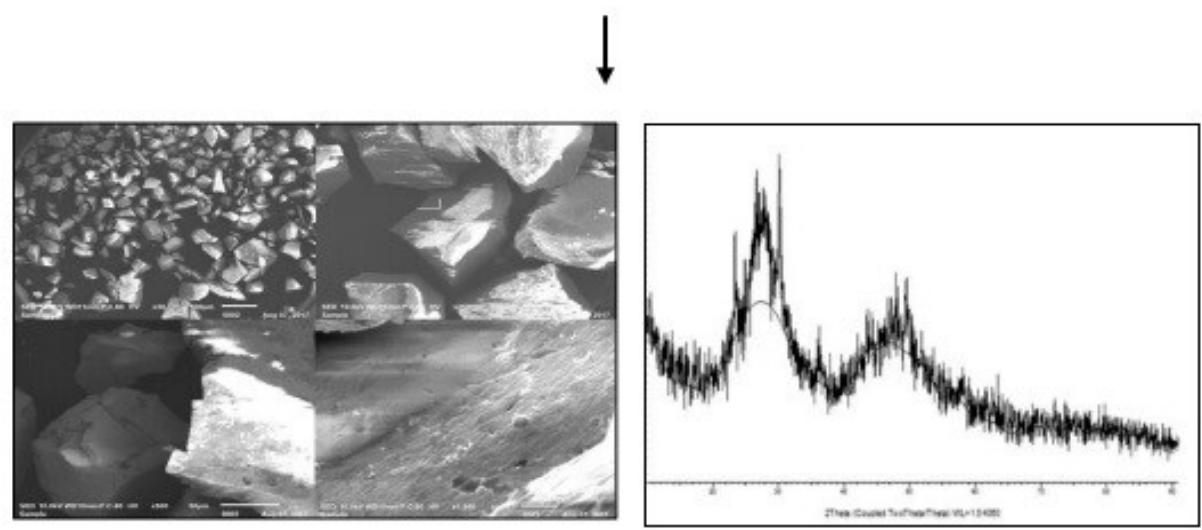

SEM

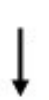

XRD

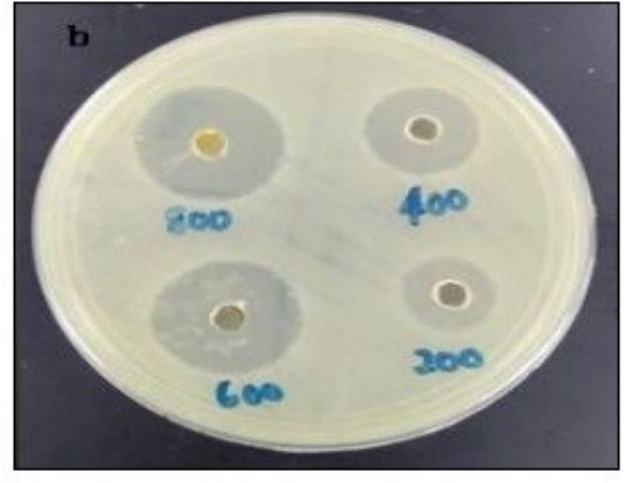

CdS nanoparticles against E.coli.

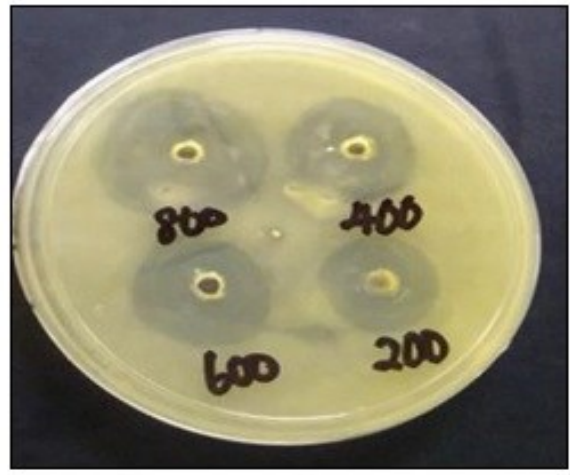

CdS nanoparticles against $\mathrm{S}$. aureus

\section{Introduction}

The integration of cadmium sulfide nanoparticles of different sizes and shapes are of great significance for their applications in various fields due to their physicochemical properties [1, 2]. Cadmium sulfide nanoparticles have found numerous applications in biomedical field as it has controllable, outstanding luminescence, continuous excitation spectrum, and narrow emission 
bands, and ease of the functionalization for tissue targeting [3-5]. It has been broadly studied in photocatalysis [6], used as biological sensors [7], and light emitting diodes [8]. However, to fulfill the demand of nanoparticles, commercially viable and environmental friendly route is needed to generate CdSNPs.

Formation of cadmium sulfide can be carried out using various techniques including, successive ionic layer adsorption and reaction, sonochemical method, microwave radiation, and sol-gel processing of polymer films containing cadmium atoms or ions with vapors of hydrogen sulfide and others $[9,10]$. Many physical and chemical methods used to produce CdSNPs with the desired characteristics. Even though these methods may productively obtain pure CdSNPs, they are generally costly, huge workforce needed, and probably risky to the biological organisms and environment. The use of an environmentally benign ingredient such as plant extracts for yielding CdSNPs offers tremendous advantages of eco-friendliness and compatibility for pharmaceutical and therapeutics practice as they do not adapt poisonous chemicals for generating a protocol. In addition, chemical approaches may leave over some toxic chemicals, enabling the absorption on the particles surface that may affect the medical functions. The green synthesis implements betterment over chemical and physical scheme as it is cheaper, ecological friendly, easier to synthesize, low applied pressure, temperature, energy and toxic chemicals.

Hereupon, the present research reports the synthesis of CdSNPs using a plant Panicum sarmentosum, reducing the cadmium (II) ions present in the solution of cadmium acetate and merged sulfide ion from the sodium sulfide solution by the cell-free aqueous extract of Panicum sarmentosum. Further, these green synthesized CdSNPs were found to be toxic against the Staphylococcus aureus and Escherichia coli.

\section{Experimental}

Preparation of plant extract

Panicum sarmentosum was collected, dried under shade, and blended into fine powder. $10 \mathrm{~g}$ of Panicum sarmentosum powder was weighed and added into $100 \mathrm{~mL}$ of distilled water. The resulting mixture was boiled in a water bath at $100{ }^{\circ} \mathrm{C}$ for $30 \mathrm{~min}$. Then, the solution was left for $1 \mathrm{~h} \mathrm{[11]}$, and filtered with Whatman No. 1 filter paper.

\section{Synthesis of cadmium sulfide nanoparticles}

10 milli mole $(\mathrm{Mm})$ aqueous solution of cadmium acetate $\left(\mathrm{CdC}_{4} \mathrm{H}_{6} \mathrm{O}_{4}\right)$ and $10 \mathrm{mM}$ aqueous solution of sodium sulfide $\left(\mathrm{Na}_{2} \mathrm{~S}\right)$ were prepared and used for synthesis of the cadmium sulfide nanoparticles. $5 \mathrm{~mL}$ of the plant extract was added to $50 \mathrm{~mL}$ of $\mathrm{CdC}_{4} \mathrm{H}_{6} \mathrm{O}_{4}$ solution for reduction into 
$\mathrm{Cd}^{2+}$ ions and $50 \mathrm{~mL}$ of sodium sulfide solution was added dropwise into the mixture to allow the $\mathrm{Cd}^{2+}$ ions gain $\mathrm{S}^{2-}$ for synthesis CdSNPs. After that, the resulting mixture placed in a rotatory orbital shaker for $12 \mathrm{~h}$ at $200 \mathrm{rpm}$, operated at $300^{\circ} \mathrm{C} .3 \mathrm{~mL}$ aliquot of the mixture was sampled after $12 \mathrm{~h}$ for monitoring. The cadmium sulfide nanoparticles (CdSNPs) obtained by Panicum sarmentosum extract were centrifuged at $10000 \mathrm{rpm}$ for $30 \mathrm{~min}$ and subsequently dispersed deionize water to get rid of any uncoordinated biological material [12].

UV-vis spectra analysis

Formation of the CdSNPs was monitored by measuring the UV-vis spectrum of the reaction medium before and after $12 \mathrm{~h}$ of incubation. UV-vis spectral analysis was carried out using UV-vis spectrophotometer thermo fisher scientific model 4001/4. The scanning range for the samples was 200-1000 nm. $100 \mathrm{uL}$ of aliquot cadmium sulfide before incubated with orbital shaker was placed in a cuvette by micropipette. Then, the absorbance was taken. The same step was repeated with the cadmium sulfide solution after $12 \mathrm{~h}$ of incubation. The band gap energy of the green synthesized CdS nanoparticles was calculated using Equation 1, as reported by Goud el al. [13];

$$
E_{g}=h c / \lambda_{\text {cutoff }}
$$

where $E_{g}$ is the band gap energy in joules of the CdS nanoparticle, planck's constant $(\mathrm{h})=$ $6.626 \times 10^{\wedge}-34$ joules sec, velocity of light $(\mathrm{c})=2.99 \times 10^{\wedge} 8$ meter $/ \mathrm{sec}$ and $\lambda_{\text {cutoff }}$ is cut-off wavelength or onset absorption wavelength. $1 \mathrm{eV}=1.6 \times 10^{\wedge}-19$ joules.

\section{$X$-ray diffraction $(X R D)$ analysis}

The obtained CdSNPs solution was purified by centrifugation at $10000 \mathrm{rpm}$ for $30 \mathrm{~min}$, followed by washing the CdSNPs pellet with distilled water to remove the bonded organic matter from the CdSNPs [14]. After drying the purified CdSNPs, the structure and composition were analyzed by XRD, SEM, and FT-IR analysis. The dried mixture of the CdSNPs was collected to determine the formation of the CdSNPs by an X-ray diffraction spectroscopy (Brucker Karlsruhe, Germany with serial number 208493 ) operated at $2 \theta$ range from $0^{\circ}$ to $100^{\circ}$.

\section{Observation of cadmium sulfide particle size}

The average particular size of the synthesized CdSNPs can be calculated using the Debye Scherrer's equation (Equation 2) [13]. 


$$
\tau=0.89 \lambda / \beta \theta
$$

where $\tau$ is the mean crystalline size, $\lambda$ is the X-ray wavelength of incident rays, $\beta$ is the width of the peak at half maximum intensity, and $\theta$ is the Bragg angle (in degrees).

\section{SEM/EDS analysis of cadmium sulfide nanoparticles}

Scanning electron microscopic (SEM) analysis was carried out using JEOL USA scanning electron microscope, model JSM-IT 100. The elemental analysis of the specimens was also assessed using the energy dispersive X-ray spectroscopey (EDS).

\section{FT-IR analysis}

The functional groups associated with cadmium sulfide nanoparticle were analyzed using the FT-IR spectroscopy (Thermo scientific with model number Iz 10). Before analyzing the samples, ZnSe crystal sample holder was cleaned and dried. A small neat nanoparticle powder was placed directly on ZnSe crystal with a spatula. Then, the knob of attenuated total reflection (ATR) was dial until the pressure read 12. FT-IR spectra were run in the absorption range of $400-4000 \mathrm{~cm}^{-1}$.

$X$-ray fluorescence (XRF) analysis

$\mathrm{XRF}$ is an analytical technique for chemical characterization. XRF analysis of the cadmium sulfide was done using the X-ray fluorescence spectroscopy (Brucker Karlsruhe, Germany with serial number 208543). The sample solution was prepared by pouring them into a plastic sample cup to ensure a flat surface to the X-ray analyzer and the sample supported over the X-ray beam. The concentrations and conversion of CdS nanoparticles were determined using the X-ray fluorescence spectrophotometer (XRF).

\section{Thermal gravimetric (TGA) analysis}

Thermal gravimetric analysis (TGA) was obtained using simultaneous thermal analysis (TGA/DSC) from Mettler Toledo, orbital shaker (Lab companion model SI-600R CdSNPs) was placed on sample pan to cover the bottom of the pan as possible. Then, a reference pan was placed together with the sample pan into the furnace. The results provided quantitative and qualitative information about the physical and chemical changes that involve endothermic or exothermic reactions.

\section{Antibacterial assays}


The antibacterial assays against Escherichia coli and Staphylococcus aureus were followed by agar well-diffusion method. Nutrient agar and nutrient broth medium were used to cultivate bacteria. Stock cultures of each bacterium were grown on to nutrient agar plates. 1-2 colonies with same morphological type were picked and cultured with streak plate method on nutrient agar dish. 3-5 well-isolated colonies with common morphological type were selected from agar plate culture and inoculated in broth culture. The fluid culture was incubated for 2-6 h at $35{ }^{\circ} \mathrm{C}$ until it reaches turbidity of the 0.5 McFarland standards. After that, swab on the agar plate with sterile cotton and let it dry. Wells were made in the agar medium using sterile cork borer; diameter of each well is 6 $\mathrm{mm}$ and the well is loaded with different concentration of stack solution such as $200,400,600,800$ and $1000 \mu \mathrm{g} / \mathrm{mL}$ of CdSNPs solution. For the control and standard antibacterial agent, methanol and amoxicillin were used, respectively. The loaded plates were incubated for $24 \mathrm{~h}$ at $35^{\circ} \mathrm{C}$. After the incubation, the formation of a zone of incubation around the well was monitored and measured in $\mathrm{cm}$; the resulted zone of inhibition indicated the antibacterial activity of CdSNPs.

\section{Results and Discussion}

\section{Synthesis of cadmium sulfide nanoparticles}

Plants hold diverse phytochemicals such as flavonoid, alkaloid, tannin, and saponins which provided vast potentials for their use as bioreductive agents in reducing cationic nanoparticle into the metallic nanoparticle [15]. It is well known that cadmium sulfide nanoparticles exhibit yellowish color in aqueous solution due to excitation of surface plasmon vibrations in cadmium nanoparticles [16, 17]. As the Panicum sarmentosum extract was mixed in the aqueous solution of the cadmium (II) ion complex and mixed with sulfide ion complex, a green colour of the solution was observed. It started to change the color from a greenish colour to dark yellow due to the reduction of cadmium (II) ion and merged with sulfide ion after 12 hours of incubation (Figure 1); which indicated the formation of cadmium sulfide nanoparticles. Panicum sarmentosum crude protein contain made them own organic functional group like $-\mathrm{OH}$ and $\mathrm{C}=\mathrm{O}$ to allow then to reducing cationic $\mathrm{Cd}^{2+}$ into the $\mathrm{CdS}$ metallic nanoparticle $[18,19]$. The biochemical reaction of cadmium acetate reacts with plant broth leads to the formation of $\mathrm{Cd}^{2+}$ and combined with $\mathrm{S}^{2-}$ from sodium sulfide. The chemical equation below explain the proposed mechanism of green synthesis of nanoparticles.

$$
\begin{gathered}
\mathrm{Cd}^{2+} \mathrm{CH}_{3} \mathrm{COO}^{-}+\text {Plant extract } \rightarrow \mathrm{Cd}^{2+}+\text { Byproduct } \\
\mathrm{Cd}^{2+}+\mathrm{Na}^{+} \mathrm{S}^{2-} \rightarrow \mathrm{CdS}+\mathrm{Na}^{+}
\end{gathered}
$$




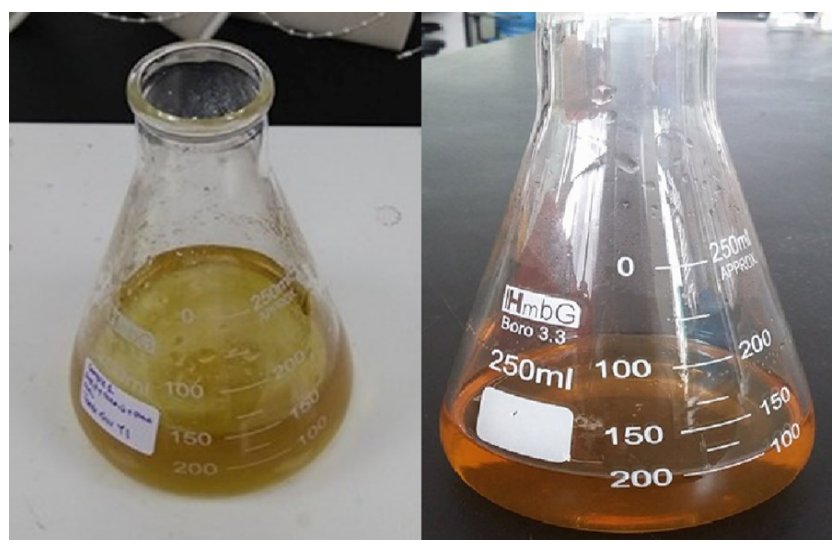

Figure 1. Resulting mixture before a) and after 12 hours b) of incubation.

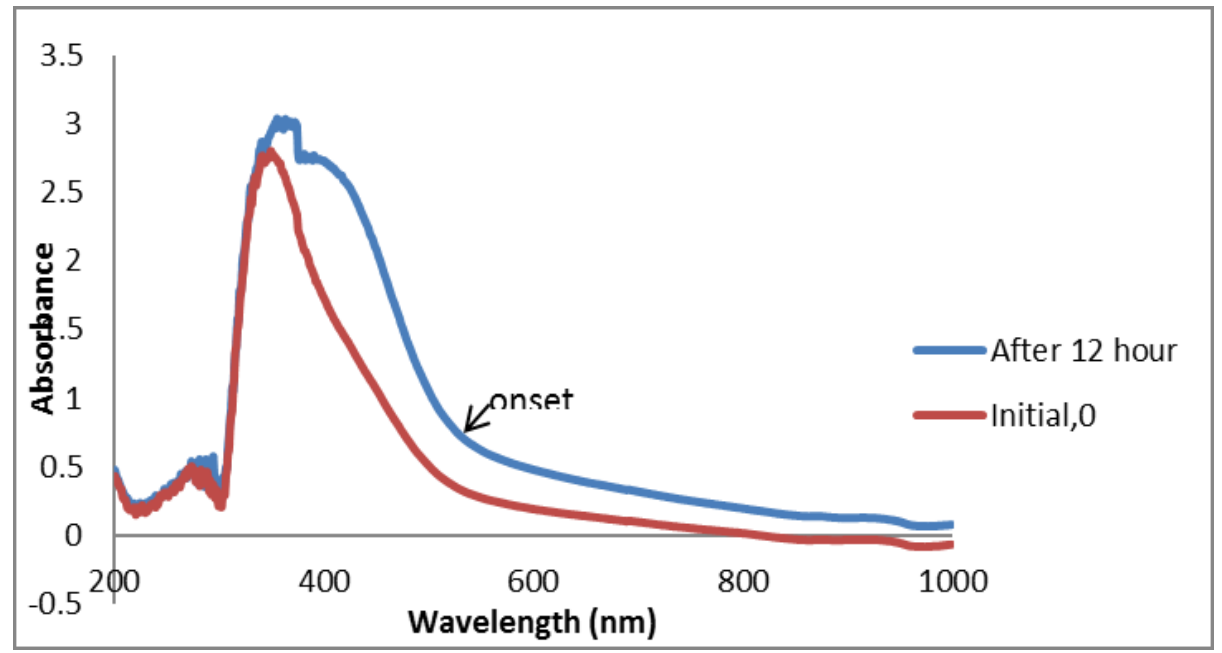

Figure 2. UV-visible Spectra of CdS nanoparticles before and after $12 \mathrm{~h}$ of incubation.

Figure 3. XRD patterns of synthesized CdS nanoparticles.

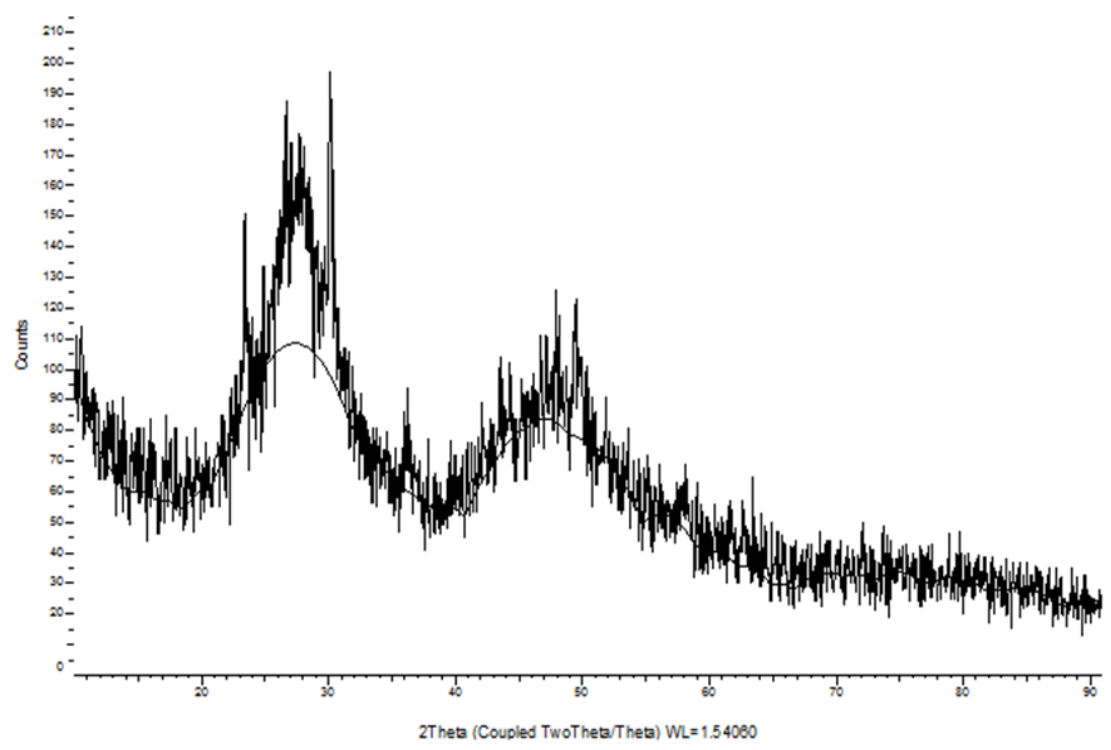




\begin{tabular}{ccccc} 
No. & $2 \theta$ value & Plane value & Element & Shape \\
1 & $26.546^{\circ}$ & 111 & CdS & Cubic \\
2 & $30.752^{\circ}$ & 200 & CdS & Cubic \\
3 & $44.047^{\circ}$ & 220 & CdS & Cubic \\
4. & $52.172^{\circ}$ & 311 & CdS & Cubic \\
\hline
\end{tabular}

There is no specific concentration of the plant extract and solution, as the concentration for both plant extract and solution increased and the reactant particles become more crowded. There is only an increased chance of the particles colliding which speed up the reaction. If the concentration of the solution is increased, there are more particles to react with the plant metabolites and fasten the reaction, vice versa.

\section{UV-vis spectra analysis}

Formation of the CdSNPs by the reduction of the $\mathrm{Cd}^{2+}$ to $\mathrm{CdS}$ during exposure to the aqueous extract of Panicum sarmentosum was followed by UV-vis spectroscopy. It is well known that cadmium sulfide nanoparticles exhibit dark yellow in water [20]. After $12 \mathrm{~h}$ of the incubation and conversion process, cadmium sulfide nanoparticle showed dark yellow color, showing the formation of cadmium sulfide nanoparticles in the solution. These colours arise due to excitation of surface plasmon vibrations in the CdS bimetallic nanoparticles [21].

Figure 2 reveals the UV-vis spectra recorded from the reaction mixture before and after $12 \mathrm{~h}$ of incubation. The UV-vis spectra of the mixture after $12 \mathrm{~h}$ of shaking showed absorption maxima between the wavelength 300-400 $\mathrm{nm}$ due to the surface plasmon resonance band of the CdS nanoparticle [22]. This confirms a blue shift from the bulk CdS which has absorption maxima of about $347 \mathrm{~nm}$. It is has been documented that UV-vis spectroscopy could be used to find band gap energy nanoparticles in aqueous suspensions. This can be attributed to the fact that that UV-visible spectroscopy has the capability to detect larger particles as well as aggregates. The band gap energy of CdS nanoparticles was estimated from the UV-visible spectrum using formula reported by Goud el al. [13] (Equation 1). The band gap energy of synthesized CdSNPs was calculated from the origin absorption of the absorbance curve, the band gap energy to be found was $2.45 \mathrm{eV}$ compared to $2.42 \mathrm{eV}$ that of bulk. The expanded band gap between the valence band and conduction band of CdSNPs was due to the quantumas confinement [13]. The enhancement of the band gap as well as the blue shift of the reflectance peak due to quantum confinement provides confirmation of the formation of CdS nanoparticles. 
$X$-ray diffraction $(X R D)$ analysis

The green synthesized CdSNPs using Panicum sarmentosum extract was further confirmed by the characteristic peaks observed in the XRD image (Figure 3). The XRD pattern exhibited four prominent peaks in the whole spectrum of $2 \theta$ value ranging from 0 to 100 . The typical XRD pattern revealed that the sample contains mixed phase (Crystalline and amorphous) structures of CdSNPs. The peaks indicate all the synthesized CdSNPs are face-centered cubic (FCC) shade. The average estimated particle size of this sample was $4.6 \mathrm{~nm}$ derived from the FWHM of the peak corresponding to (111) planes. The crystal system of diffract eva software from Bruker presented this CdSNPs was the cubic shape. The result from diffraceva software also revealed that $31.7 \%$ of the nanoparticles were crystalline and $68.3 \%$ were amorphous.

\section{SEM and EDS observation of cadmium sulfide nanoparticles}

The structural view of the synthesized cadmium sulfide nanoparticles using Panicum sarmentosum extract under the scanning electron microscope (Figure 4) shows the high density CdNPs synthesized by the Panicum sarmentosum extract which further confirmed the development of CdSNPs nanostructures. The EDS pattern of CdS nanoparticles using Panicum sarmentosum extract (Figure 5) depicted the element composition generated nanoparticles. The elemental composition of green synthesis of CdS nanoparticles was determined by EDS analysis. As depicted in Figure 5, a strong signal of cadmium atom was found. Then, strong signals of sulfur were also found. This confirmed the presence of elemental cadmium and sulfur in the sample and supporting the hypothesis that cadmium acetate is reduced by Panicum sarmentosum leaf extract. In addition, the EDS spectrum also showed signal for carbon (C), oxygen (O) and nitrogen (N), illustrating the existence of macromolecule from a plant as stabilizing agent on the surface of CdS nanoparticles.

\section{FT-IR analysis}

FT-IR analysis was employed to characterize the Panicum sarmentosum and CdSNPs (Figure 6). FT-IR spectra of the plant powder and resulting pellet are depicted in Figure 6. Absorbance bands in Figure 6a (Resulting pellet) the absorbance bands are observed in the region of $400 \mathrm{~cm}^{-1}$ to 4000 $\mathrm{cm}^{-1}$ are $3232.34 \mathrm{~cm}^{-1}, 2918.46 \mathrm{~cm}^{-1}, 2116.36 \mathrm{~cm}^{-1}, 1915.41 \mathrm{~cm}^{-1}, 1627.94 \mathrm{~cm}^{-1}, 1535.33 \mathrm{~cm}^{-1}$, $1389.72 \mathrm{~cm}^{-1}, 1232.49 \mathrm{~cm}^{-1}, 1010.20 \mathrm{~cm}^{-1}, 682.40 \mathrm{~cm}^{-1}, 663.13 \mathrm{~cm}^{-1}$ and $613.84 \mathrm{~cm}^{-1}$. These absorbance bands are known to be associated with the stretching vibrations for alcohol or phenol $\mathrm{O}-\mathrm{H}$ stretch, $\mathrm{C}-\mathrm{H}$ (of alkanes, alkyl groups), $-\mathrm{C} \equiv \mathrm{C}$ - (alkynes), $-\mathrm{C}=\mathrm{O},-\mathrm{C}=\mathrm{N},-\mathrm{C}=\mathrm{C}-$ and $-\mathrm{C}-\mathrm{Cl}$, respectively [23]. Figure 6 depictes no significant difference between the peaks of plant material and the cadmium sulfide nanoparticle, it indicated that the synthesized CdS nanoparticle from the 
Panicum sarmentosum extract capped by proteins and mineral which contain functional groups of alkanes, alcohols, alkyl, phenol and alkyl halides.

Using FT-IR spectroscopy of CdSNPs synthesized in plant extracts, it has been demonstrated that plant secondary metabolites are associated with CdSNPs. These metabolites are a class of diverse organic polymers synthesized in plants, which display strong antioxidant activity. CdSNPs get associated with the antioxidant agents and enables to avoid further oxidation.

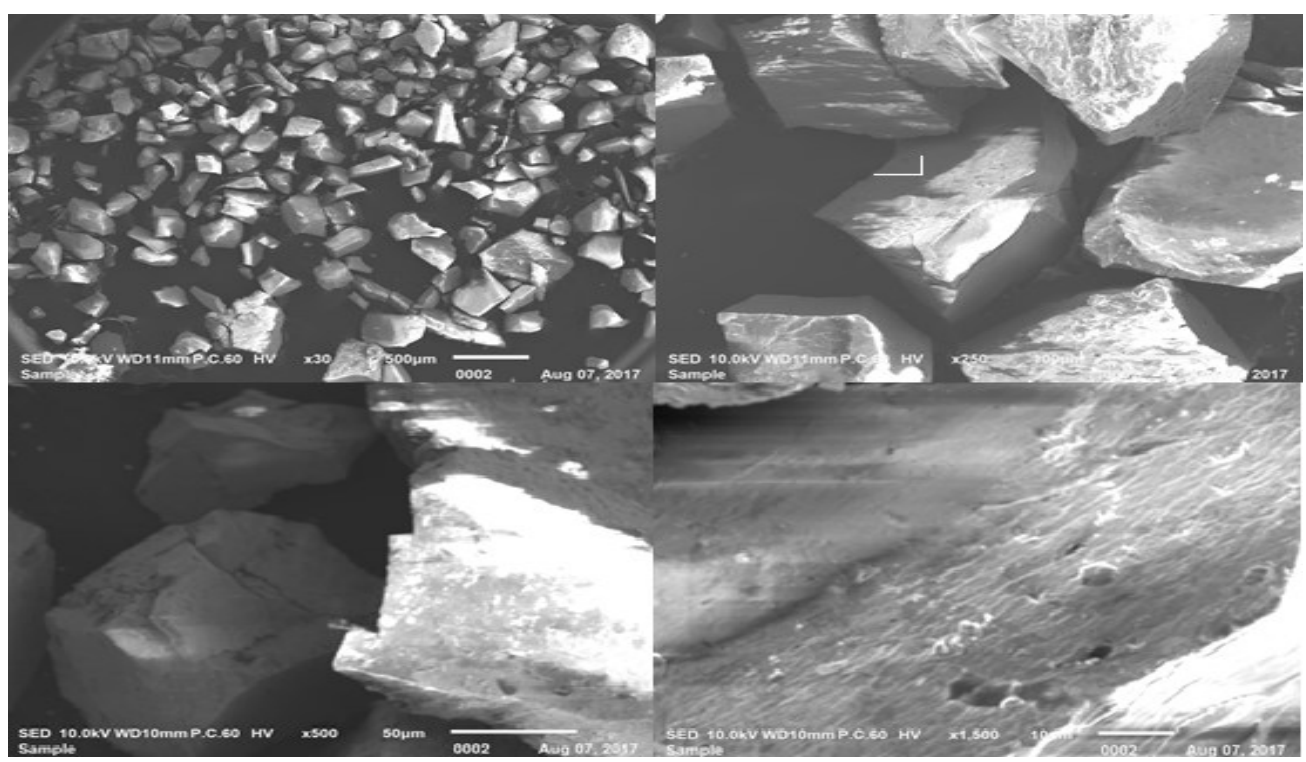

Figure 4. SEM image of CdS nanoparticles under magnification x30, x250, x500 and x1500.

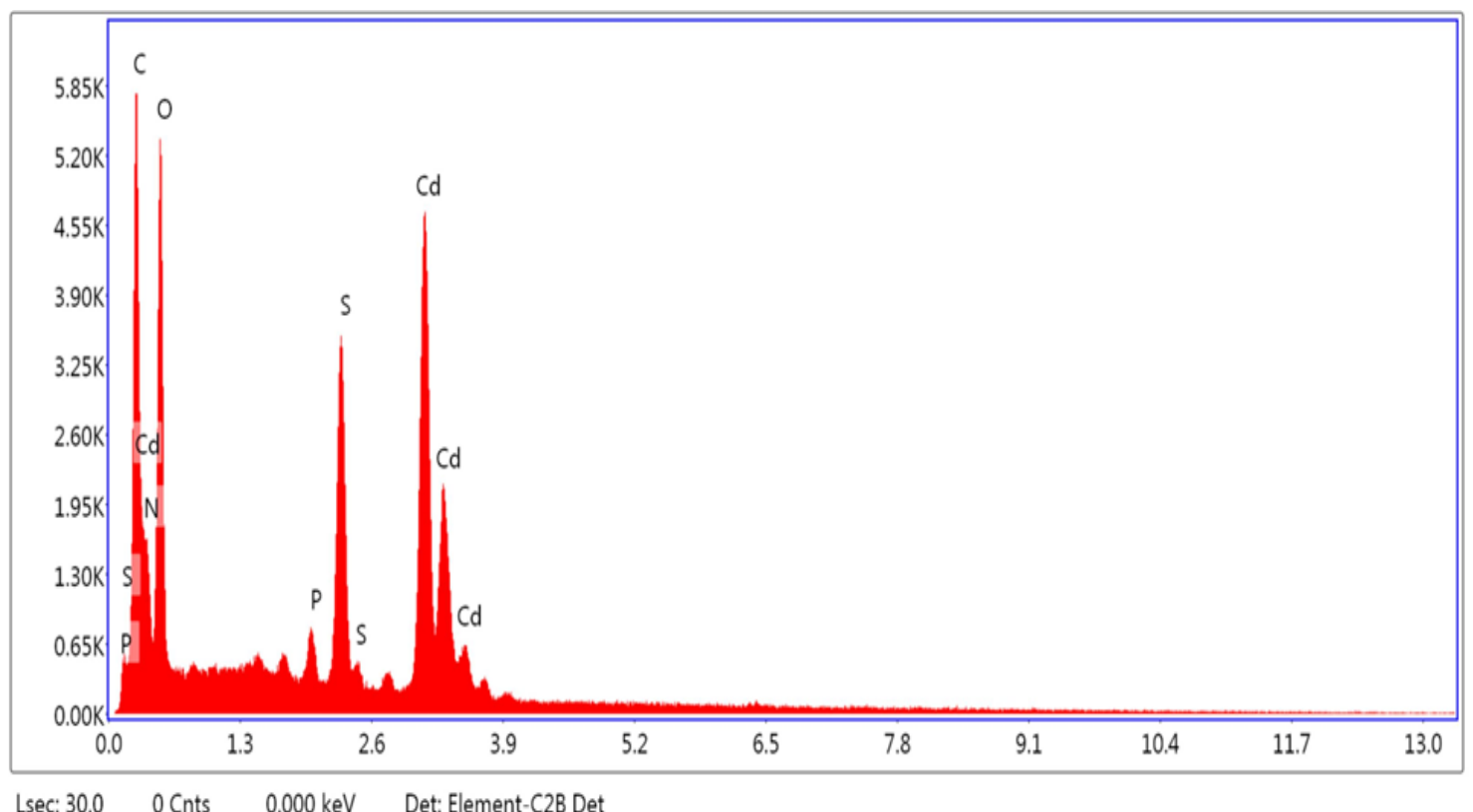




\begin{tabular}{rccc} 
Element & Weight \% & Atomic \% \\
& $\mathrm{C}$ & 19.47 & 33.62 \\
$\mathrm{~N}$ & 6.25 & 9.26 \\
$\mathrm{O}$ & 35.13 & 45.52 \\
$\mathrm{P}$ & 1.34 & 0.90 \\
$\mathrm{~S}$ & 8.08 & 5.22 \\
$\mathrm{Cd}$ & 29.73 & 5.48 \\
\hline
\end{tabular}

Figure 5. EDS pattern of cadmium sulfide synthesized using Panicum sarmentosum extract.

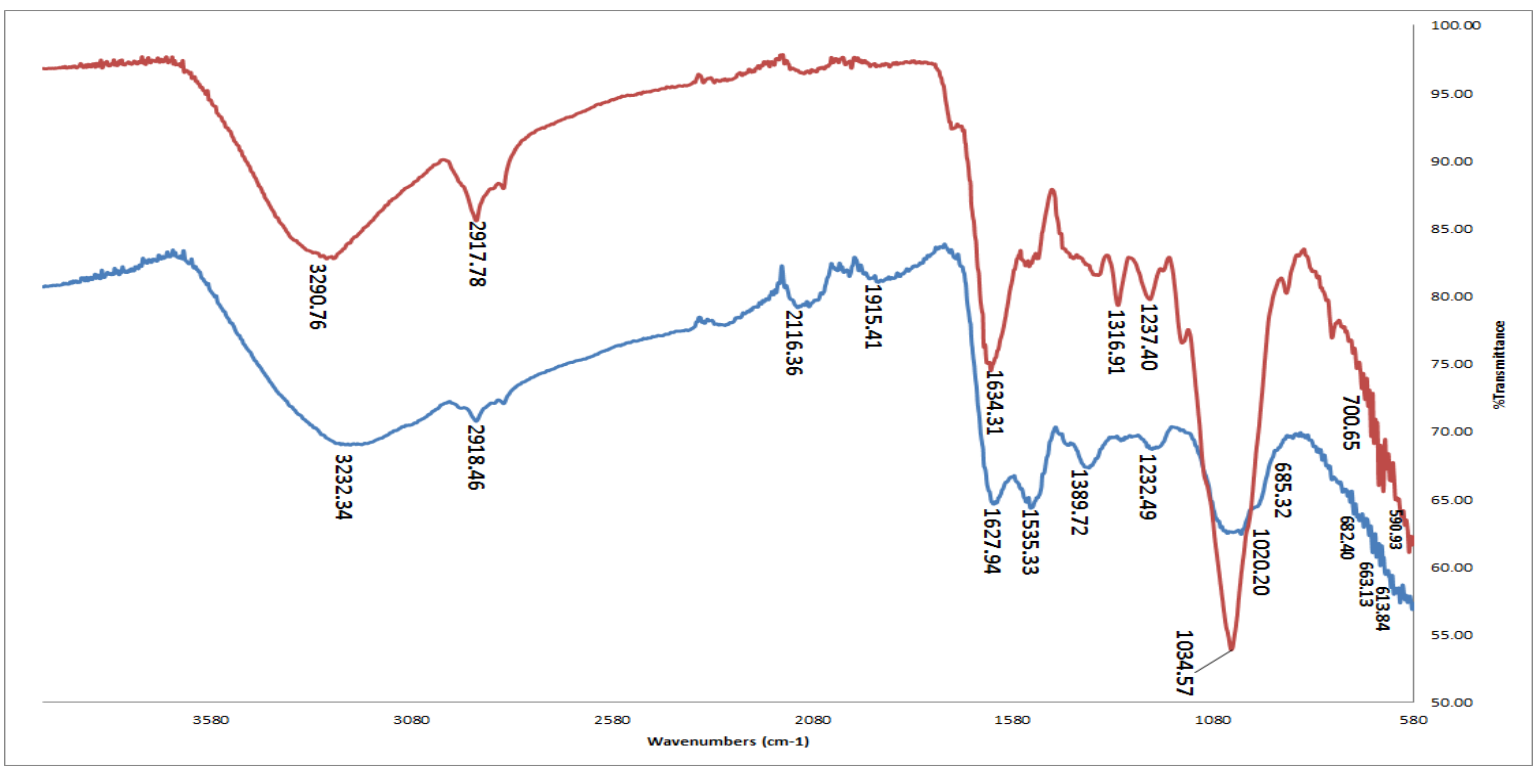

Figure 6. FT-IR spectrum of CdSNPs treated a) Panicum sarmentosum and b) Panicum sarmentosum.

X-ray fluorescence (XRF) analysis

The elemental composition of the green-synthesized CdSNPs was also studied using X-ray fluorescence spectrometer (Figure 7). It reveals the clear elemental composition profile of the green-synthesized CdSNPs. The elements existed in the synthesized CdSNPs are cadmium (Cd), sulfur $(\mathrm{S})$, potassium $(\mathrm{K})$, calcium $(\mathrm{Ca})$, phosphorus $(\mathrm{P})$, chlorine $(\mathrm{Cl})$, sodium $(\mathrm{Na})$, silver $(\mathrm{Ag})$, stannum (Sn), barium (Ba) and antimony (Sb). The presence of cadmium and sulfur atom again confirmed the formation of CdSNPs. The detected element other than $\mathrm{Cd}$ and S come from plant itself where the synthesized nanoparticles capping with plant material which supported the result of FT-IR analysis.

Thermal gravimetric (TGA) analysis 
TGA is a technique to determine the amount of weight change of a material, either as a function of increasing temperature over times, or isothermally as a function of time, in an atmosphere of nitrogen, helium, air, other gas, or in vacuum. The weight change of a material can be used to evaluate thermal stability and material characterization. TGA of the synthesized CdSNPs and plant material provided the information on the thermal decomposition profile of respective components that can be used to evaluate the thermal stability of a CdSNPs. The range of temperature below 100 ${ }^{\circ} \mathrm{C}$, both of the samples had an initial small amount of weight loss that indicated to the evaporation of water loosely bound to the surfaces of the samples. The weight loss of both samples stared when the temperature reached $50{ }^{\circ} \mathrm{C}$. Both curves of the samples, a single noticeable decomposition associated with progressive weight loss were marked. The synthesized CdSNPs were observed more thermally stable than it synthesizing raw material. This is due to the fact that only little slope in the CdSNPs TGA trace and corresponded with the negligible mass loss. The residue remained for the CdSNPs was $3.7 \mathrm{mg}$ which is approximately $55 \%$ of the original weight (6.7 $\mathrm{mg}$ ). The remaining weight of the plant material was $2.2 \mathrm{mg}(33.1 \%$ from $6.7 \mathrm{mg})$. The lower weight loss of CdSNPs revealed that it is more thermally stable than raw material. The CdSNPs decomposed in two steps in a lower rate, first were degraded speedily and last reside CdSNPs residue. The Panicum sarmentosum decomposed in three steps, first and second were rapidly, then slows as the last remaining plant decomposes. The experimental result showed 3.8\% and $40.7 \%$ degradation of synthesized CdSNPs and 3.5\%, 48.4\% and 14.8\% degradation of Panicum sarmentosum during first, second and third step, respectively. The first pyrolysis of CdSNPs was due to the removal of moisture (hydrogen and oxygen molecules) and in the second pyrolysis, removal the capping plant material occurs. The thermally stable of CdSNPs could be due to the ionic bond between $\mathrm{Cd}^{2+}$ and $\mathrm{S}^{2}$. This is because of strong electrostatic forces of attraction present between ions. Thus, CdSNPs require a lot of thermal energy to overcome these force of attraction.

Figure 7. XRF data of cadmium sulfide nanoparticles treat with Panicum sarmentosum

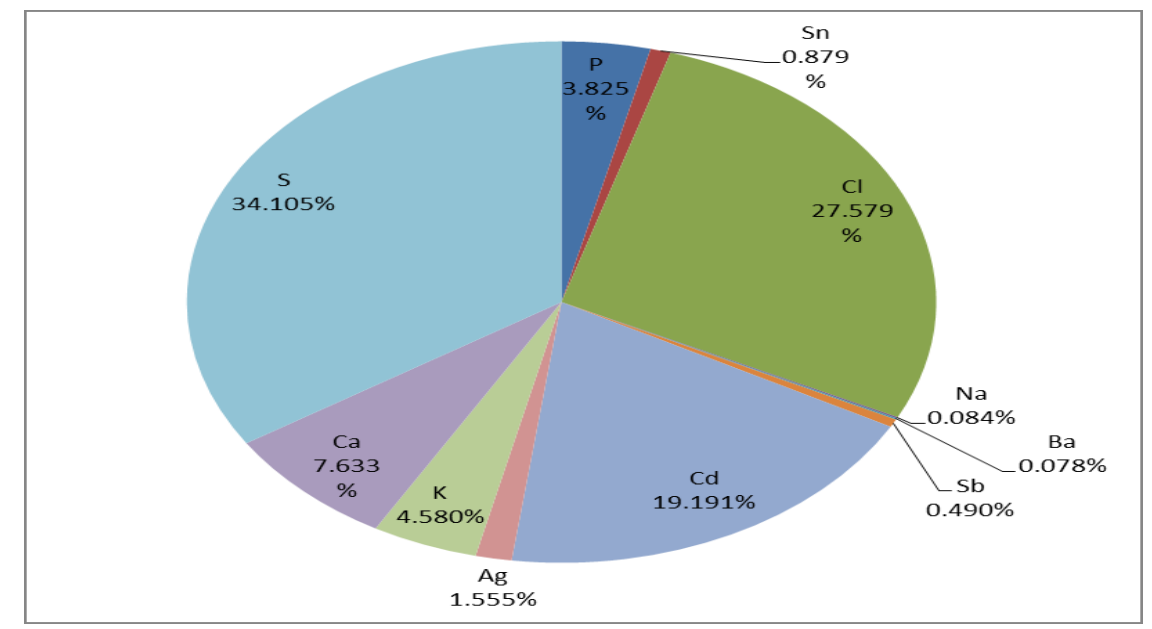




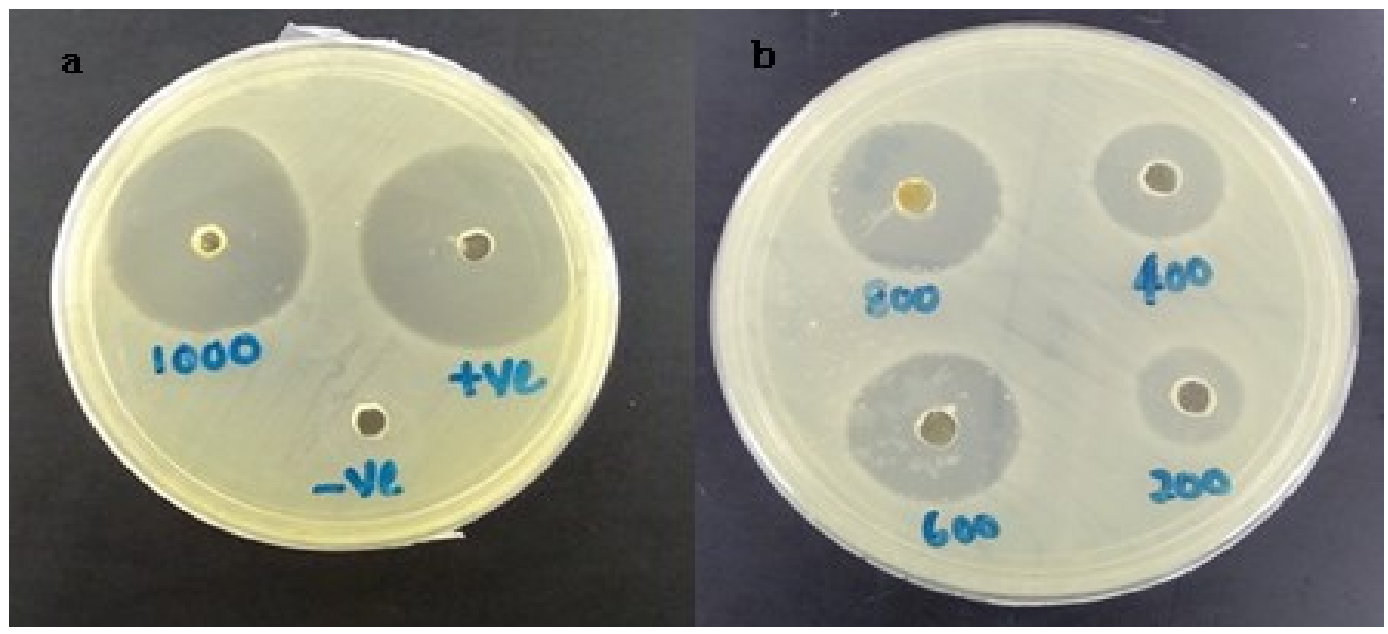

Figure 8. Showing antibacterial activity of CdS nanoparticles of Panicum sarmentosum againts Escherichia coli a) antibacterial activity of positive control (Amoxicilin), negative control (methanol) and $1000 \mu \mathrm{g} / \mathrm{mL}$ of CdS nanoparticles b) antibacterial activity $800 \mu \mathrm{g} / \mathrm{mL}, 600 \mu \mathrm{g} / \mathrm{mL}$, $400 \mu \mathrm{g} / \mathrm{mL}, 200 \mu \mathrm{g} / \mathrm{mL}$ of CdS nanoparticles

\section{Antibacterial assays}

The CdSNPs synthesized by green route were found toxic against Escherichia coli (Figure 8) and Staphylococcus aureus (Figure 9). CdSNPs exhibited antibacterial activity against Escherichia coli and Staphylococcus aureus in various concentrations as it showed a clear inhibition zone. It was found out that with an increasing concentration of the nanoparticle suspension the antimicrobial property increased and the larger zone of inhibition of the bacteria (Figure 10). In addition, gramnegative (Escherichia coli) bacteria were found more resistant to CdSNPs than gram-positive bacteria (Staphylococcus aureus) as observed in $1000 \mu \mathrm{g} / \mathrm{mL}, 800 \mu \mathrm{g} / \mathrm{mL}, 600 \mu \mathrm{g} / \mathrm{mL}, 400 \mu \mathrm{g} / \mathrm{mL}$ and $200 \mu \mathrm{g} / \mathrm{mL}$ of CdSNPs suspension. This is because of the gram-negative outer membrane comprises a complex lipopolysaccharide (LPS) whose lipid portion acts as an endotoxin which does not absorb foreign materials that surround it. The inhibitory action of cadmium sulfide nanoparticle on bacterial cells is related to the strong interaction of $\mathrm{CdS}$ with thiol groups present in key respiratory enzymes in bacteria. The positive charge from $\mathrm{CdS}$ nanoparticles combine electrostatically with the negative charge of proteins on the surface of the bacterial cell and pass through the membrane to change the internal composition of the bacteria.

\section{Conclusion}

In the present study, we have found that leaves can be a good source for the synthesis of CdSNPs. This green chemistry approach toward the synthesis of CdSNPs is acquired with many benefits such 


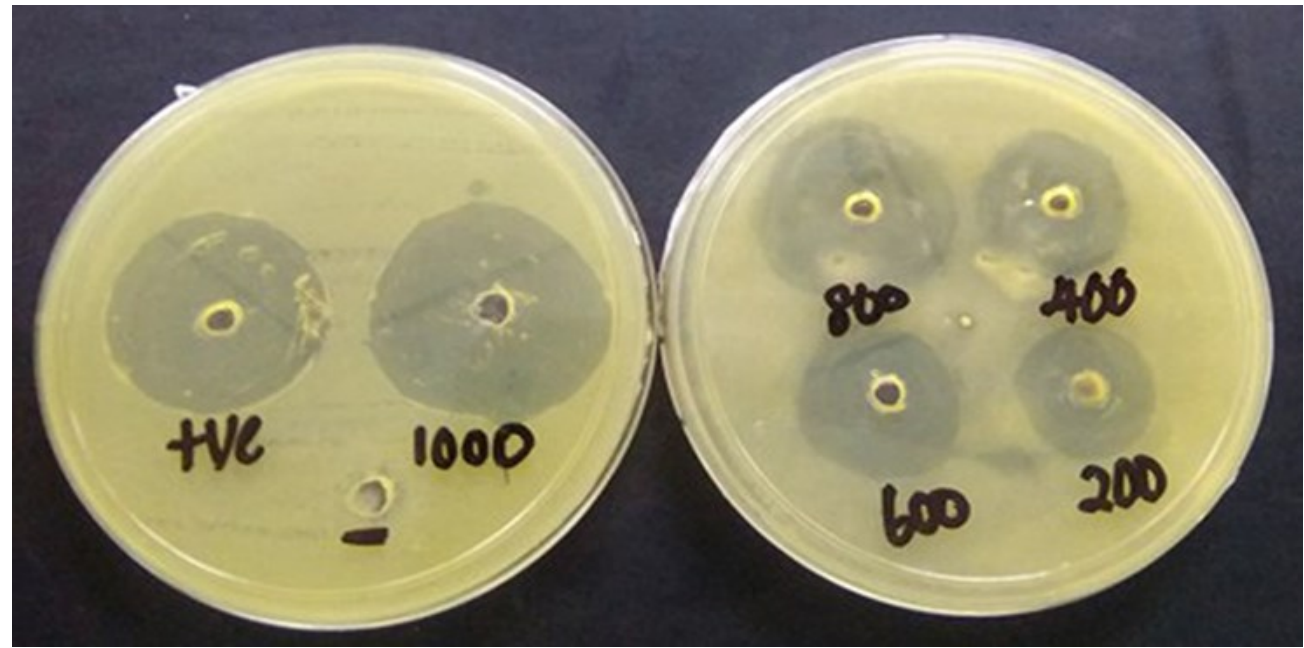

Figure 9. Showing antibacterial activity of CdS nanoparticles of Panicum sarmentosum againts Staphylococcus aureus a) antibacterial activity of positive control (Amoxicilin), negative control (methanol) and $1000 \mu \mathrm{g} / \mathrm{mL}$ of CdS nanoparticles b) antibacterial activity $800 \mu \mathrm{g} / \mathrm{mL}, 600 \mu \mathrm{g} / \mathrm{mL}$, $400 \mu \mathrm{g} / \mathrm{mL}, 200 \mu \mathrm{g} / \mathrm{mL}$ of CdS nanoparticles

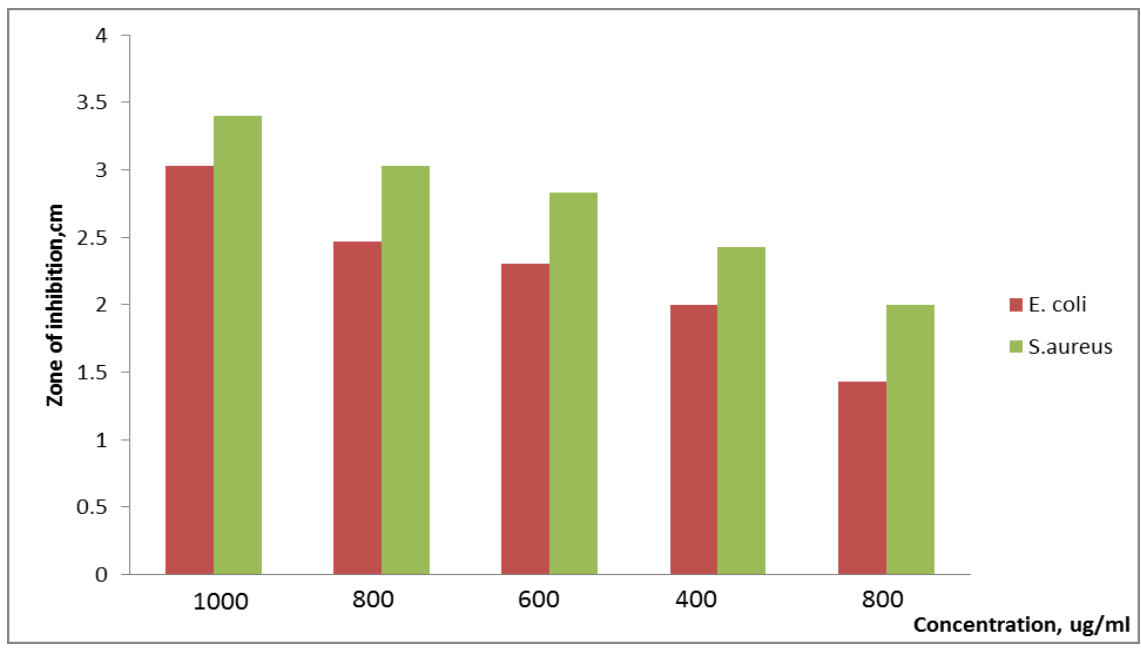

Figure 10. Showing graph of inhibition zone (cm) for Escherichia coli and Staphylococcus aureus against CdSNPs concentration $(\mu \mathrm{g} / \mathrm{mL})$

as ecologically friendly, cheap, and easy to generate. The applications of such eco-friendly generated nanoparticles as antibacterial agents can make this method potentially exciting for the large-scale synthesis of other inorganic nanomaterials. The toxicity studies of CdSNPs on Escherichia coli and Staphylococcus aureus can open new avenues for the preparation of antibacterial agents.

\section{Disclosure Statement}

No potential conflict of interest was reported by the authors. 


\section{References}

[1]. Murray C.B., Kagan C.R., Bawendi M.G. Annu. Rev. Mater. Sci., 2000, 30:545

[2]. Hu J., Odom T.W., Lieber C.M. Acc. Chem. Res., 1999, 32:435

[3]. Kar S., Chaudhuri S. Synth. React. Inorg. Metal-Organic Nano-Metal Chem., 2006, 36:289

[4]. Yang G., Qin D., Du X., Zhang L., Zhao G., Zhang Q., Wu J. J. Alloy. Compd., 2014, 604:181

[5]. Tayade R.J. Natarajan T.S., Bajaj H.C. Industrial \& Engineering Chemistry Research, 2009,

48:10262

[6]. Yang H., Huang C., Li X., Shi R., Zhang K. Mater. Chem. Phys., 2005, 90:155

[7]. Pardo-Yissar V., Katz E., Wasserman J., Willner I. J. Am. Chem. Soc., 2003, 125:622

[8]. Zhao J., Bardecker J.A., Munro A.M., Liu M.S., Niu Y., Ding I.K., Luo J., Chen B., Jen A.K.Y., Ginger D.S. Nano Lett., 2006, 6:463

[9]. Raziya S., Durga B., Rajamahanthe S.G., Govindh B., Annapurna N. Int. J. Adv. Technol. Eng. Sci., 2016, 4:220

[10]. Xaba T., Moloto M.J., Moloto N. Mater. Lett., 2015, 146:91

[11]. Naseem T., Farrukh M.A. Journal of Chemistry, 2015, 2015:7 page

[12]. Prasad K.S., Amin T., Katuva S., Kumari M., Selvaraj K. Arabian J. Chem., 2017, 10:S3929

[13]. Goud B.S., Suresh Y., Annapurna S., Singh A.K., Bhikshamaiah G. Mater. Today: Proce., 2016, 3:4003

[14]. Rao M.D., Pennathur G. Mater. Res. Bull., 2017, 85:64

[15]. Hatti-Kaul R., Törnvall U., Gustafsson L., Börjesson P. Trends biotech., 2007, 25:119

[16]. Bai H.J., Zhang Z.M., Guo Y., Yang G.E. Colloid. surfaces B: Biointerfaces, 2009, 70:142

[17]. Isarov A.V., Chrysochoos J. Langmuir, 1997, 13:3142

[18]. Wilson J.R., Brown R.H. Crop Sci., 1983, 23:1148

[19]. Allison S.D., Chang B., Randolph T.W., Carpenter J.F. Arch. Biochem. Biophys., 1999, 365:289

[20]. O'Brien P., Saeed T. J. Crystal growth, 1996, 158:497

[21]. Barnes W.L., Dereux A., Ebbesen T.W. Nature, 2003, 424:824

[22]. Baset S., Akbari H., Zeynali H., Shafie M. Digest Journal of Nanomaterials and Biostructures, 2011, 6:709

[23]. Silverstein R.M., Bassler G.C., Morrill T.C., Spectrometric Identification of Organic Compounds. $4^{\text {th }}$ ed. New York: John Wiley and Sons, 1981; p x +419

How to cite this manuscript: Irshad Ul Haq Bhat*, Yong Sin Yi. Green synthesis and antibacterial activity of cadmium sulfide nanoparticles (CdSNPs) using panicum sarmentosum. Asian Journal of Green Chemistry, 3(4) 2019, 455-469. DOI: 10.33945/SAMI/AJGC.2019.4.3 